\title{
DESIGN AND SIMULATION OF A LIGHT MANIPULATOR USING ADAMS VIRTUAL PROTOTYPE
}

\author{
Pengfei $\mathrm{Li}^{1 *}$, Xiang Cao ${ }^{1}$ \\ 1Zhengzhou Railway Vocational and Technical College, Zhengzhou, 451460, Henan, China. \\ ${ }^{*}$ Corresponding author: PengFei Li \\ Email: pengfei664@126.com
}

\begin{abstract}
In order to study the structure of light manipulator and find the method to improve the structure design of the manipulator, the three-dimensional complex simulation model of the light manipulator is established with the help of the most commonly used mechanical system analysis software, and the movement process and the actual specific working conditions of the manipulator are simulated. In addition, the simulation results are compared with the actual working conditions to study the light manipulator and improve its structure. The method used is based on ADAMS (automatic dynamic analysis of mechanical systems). Through simulation, the virtual prototype is constructed, the model describing the structure of the light manipulator is established, and the actual motion condition of the manipulator is analysed. The findings show that: the threedimensional model of the light manipulator is built in ADAMS, and the hinge point and the length of the rod are parameterized. The end output power of the light manipulator is taken as the optimization objective to design the structure. The maximum power of the redesigned light manipulator is higher than that of the conventional manipulator, and the effect of the new design structure is obviously superior to the design in the conventional methods. Therefore, if this creation is applied to the actual production and life, it will be able to reduce the energy consumption to the greatest extent and improve the production efficiency. Thus, the structure of the light manipulator in accordance with ADAMS virtual prototype technology not only has a dramatic practical significance, but also has reference for the subsequent research on the improvement of the structure of the manipulator for various purposes.
\end{abstract}

Keywords: ADAMS; Virtual Prototype; Light Manipulator; Structure Design; Simulation.

\section{Introduction}

After entering the 21st century, RT (robot technology), also known as AIT (artificial intelligence technology), attracts worldwide attention [1, 2]. Almost all the countries are striving to conduct studies in the field, and the robot also becomes the main battlefield of scientific and technological competition among countries. Therefore, people will enter the era of robots in the near future, and the relationship with artificial intelligence is increasingly close [3].

The research of robots in China begins in the 1990s. The research of industrial robots is earlier than that of service robots, but it is still later than that of western countries. Up to now, the research of robot technology in China is over 30 years, and all kinds of robots also enter into life. In manufacturing automation, industrial robots are used to deal with machinery manufacturing tasks, while service robots can provide life service for the public. Various robots, such as sweeping robots in families, automatic meal delivery robots in hotels, police security robots, business operators in scenic spots and banks, greatly enrich the lives, promote the production, and maintain the social order $[4,5]$. In terms of the shapes of various robots, the industrial robots with light manipulator are appropriate for human production activities, and are widely used in various complex working conditions. Therefore, light manipulator robot plays an important role in human production and life. The robot applied should bear the following characteristics: simple structure, light weight, flexible motion, variable trajectory, high reliability, and safety [6, 7].

ADAMS is a virtual prototype analysis software developed by MSC in the United States. The software uses graphical interactive interface, parts library, constraint library and other databases to establish a fully parameterized mechanical system, and uses the solver based on the dynamics theory of multi-rigid body system to analyse the kinematics and dynamics of the geometric model of the virtual mechanical system, obtaining the displacement, velocity and reaction force curves of the machine and other parameters [8]. ADAMS software can be used to predict the mechanical performance of the mechanical system, including the range of motion, 
the peak load, and the input load. Users can utilize the software to analyse the statics, kinematics and dynamics of the virtual prototype. And the software can provide the external program interface for the secondary development scheme of the special types of virtual prototype in different industries $[9,10]$.

MATLAB (Matrix Laboratory) is a commercial mathematics software developed by MathWorks in the United States. Its functions are as follows: data visualization, numerical analysis, simulation and numerical calculation through advanced technology computing language and interactive development environment. It mainly includes MATLAB and Simulink [11]. MATLAB has excellent numerical calculation ability, unique matrix calculation, function analysis, image drawing and the connection with other programming development languages, which makes it widely used in signal processing, simulation, mathematical modelling and image processing. MATLAB software is used here to simulate and predict the calculation results of the motion trajectory of the manipulator [12-14].

The structure of the light manipulator is designed, and the mechanical system simulation analysis is carried out by using ADAMS and MATLAB software. The relevant simulation model is established, and the actual working conditions of the manipulator model are comprehensively and systematically discussed. ADAMS virtual prototype simulation is applied to the structural design and improvement of light-weight manipulator, the hinge point and rod length requirements in the operation process are analysed, and the light-weight manipulator suitable for the needs is designed, which has practical significance for the current mechanical research and provides foundation for the future theoretical research.

\section{Methods and Simulation Experiments 2.1 ADAMS Theory}

(1) Basic information of ADAMS software

ADAMS software is a virtual prototype analysis system developed by MDI in America. It can be used to analyse dynamics and kinematics of virtual mechanical system, and provides secondary development interface for special virtual prototype. In the ADAMS simulation, the actual performance indexes such as environment and mechanical properties need to be input into the system to predict the performance, collision point, range of motion and other data of mechanical system are obtained. ADAMS software has special data environment such as interface, part library and constraint database. The Lagrange equation is used to analyse the multi rigid body system, and a new dynamic equation of virtual mechanical system is established to obtain the output displacement, velocity and acceleration.
The data of collision detection, motion range and load peak can be obtained by simulation. The simulation analysis of virtual mechanical system can be easily carried out by ADAMS software users, and the results of static, dynamic and kinematic analysis are obtained. The program interface can also provide the secondary development for users. Currently, ADAMS software has two system versions: UNIX version and Windows NT/2000 version. The software used is ADAMS 12.0 software of Windows 2000 version $[15,16]$.

(2) Software module

The basic composition of ADAMS software includes five modules: basic module, expansion module, interface module, professional domain module and toolbox. The basic module can be used to simulate and test the general mechanical system, while the specialized module can be used to model and simulate the mechanical application problems in the specific industrial environment. Users can use the software to carry out mechanical simulation, establish and test the performance of virtual prototype system on computer, realize the technical requirements of real-time simulation, and understand the actual motion performance of complex mechanical system. MD Adams and MD NASTRAN complement each other in the enterprise MSC SimEnterprise simulation environment, which can provide a complete engineering simulation analysis environment for complex mechanic, while SimEnterprise simulation environment is a more completely integrated simulation and analysis method. MD Adams can play a fully role in the simulation of motion structure coupling, and the output results of Adams model are transmitted to Nastran through bi-directional integration of MD Nastran for more detailed NVH analysis or stress recovery, and life / damage calculation [17].

\subsection{Kinematics Calculation of Manipulator}

The calculation of the trajectory of the manipulator is the basis of analysing the motion control of the mechanical part. The following is to calculate the trajectory from the forward and reverse kinematics of the manipulator, and verify the results by using MATLAB. Pose description is a comprehensive description method of position and attitude, which is used to study the trajectory and the actual working condition of manipulator. In three-dimensional space, if the set state of an object needs to be determined, not only is the position of the object determined, but also the orientation and position of the object are accurately described. The process of quantitatively describing the position and orientation of the manipulator. is known as the description of the pose of the object $[18,19]$. 
(1) Location description

In the traditional Cartesian coordinate system, the following vectors are used to represent the positions ${ }^{\wedge} P$ in the three-dimensional space, as shown in equation 1 , where $P$ is the position vectors and $P_{x}, P_{y}, P_{z}$ are the vector components of $P$ on the coordinate axes $x, y$ and $z$ respectively, as shown in Figure 1 , which is the three-dimensional vector space.

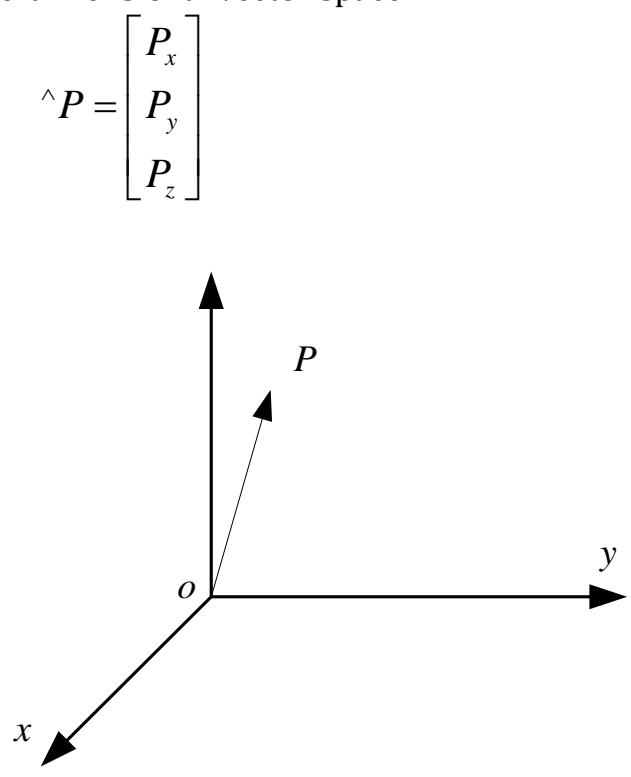

Figure. 1 Location description

After the position of a connecting rod is determined, it is necessary to clarify the relative position relationship of each connecting point, determine the orientation, and establish an independent Cartesian coordinate system on each connecting point. And then the mutual orientation description between the connecting points is described by the following matrix equation (2) [20, 21].

$$
{ }_{B}^{A} R=\left[{ }^{A} X_{B}{ }^{A} Y_{B}{ }^{A} Z_{B}\right]=\left[\begin{array}{c}
n_{x} n_{y} n_{z} \\
o_{x} o_{y} o_{z} \\
a_{x} a_{y} a_{z}
\end{array}\right]
$$

Assuming that the rectangular coordinate system $B$ is fixedly connected with the rigid body $B$, the position of the rigid body $B$ is determined by using the position change of the coordinate system $B$ compared with the coordinate system $A .{ }_{B}^{A} R$ represents the matrix composed of three main vectors of the available coordinate system relative to the cosine of the three main axes of the coordinate system, which is called the rotation matrix.

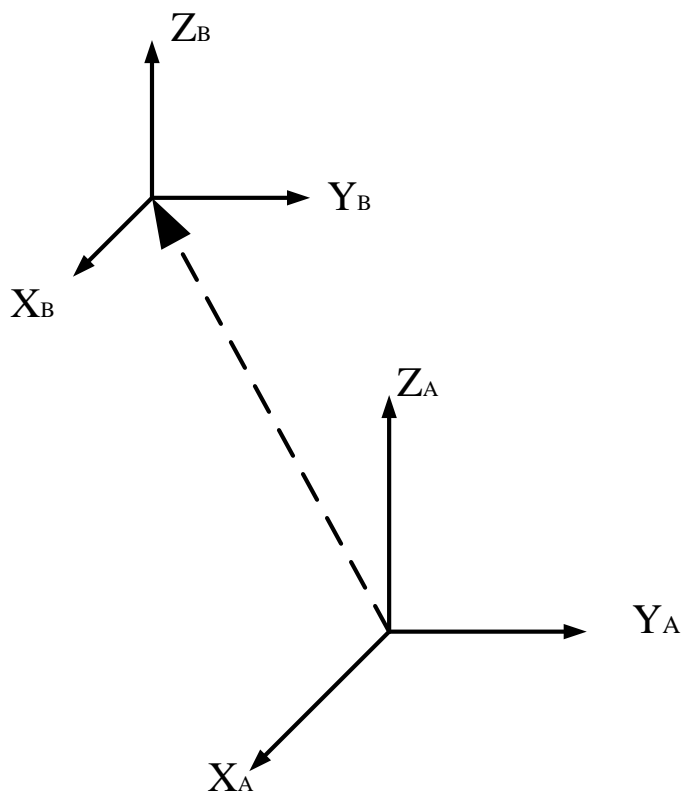

Figure. 2 Orientation description

Because ${ }_{B}^{A} R$ is an orthogonal matrix, it has six constraints, which are expressed by the following equations (3) and (4):

$$
\begin{aligned}
& { }^{A} X_{B} \cdot{ }^{A} X_{B}={ }^{A} Y_{B} \cdot{ }^{A} Y_{B}={ }^{A} Z_{B} \cdot{ }^{A} Z_{B}=1 \\
& { }^{A} X_{B} \cdot{ }^{A} Y_{B}={ }^{A} Z_{B} \cdot{ }^{A} Y_{B}={ }^{A} Z_{B} \cdot{ }^{A} X_{B}=0
\end{aligned}
$$

The rotation matrix is orthogonal and the condition that ${ }_{B}^{A} R^{-1}={ }_{H}^{B} R^{T}$ and $\left|{ }_{B}^{A} R\right|=1$ are equal to 1 is satisfied. When the manipulator is timecounted, the conversion matrix is used to intuitively represent the rotation direction of each joint of the machine, the reference coordinate system, and the converted coordinate system. And it is expressed in equation (5) or (6) [22].

$$
\begin{aligned}
& { }_{B}^{H} R={ }_{H}^{B} R^{-1} \\
& { }_{H}^{B} R={ }_{B}^{H} R^{-1}
\end{aligned}
$$

Rotate the above calibration around $x$ axis. And the angle rotated and the transformation matrix is as follows [23].

$$
R(x, \alpha)=\left[\begin{array}{ccc}
1 & 0 & 0 \\
0 & c \alpha & -s \alpha \\
0 & s \alpha & c \alpha
\end{array}\right]
$$


The matrix of rotation angle $\beta$ around $Y$ axis is the change matrix, as shown in equation (8).

$$
R(y, \beta)=\left[\begin{array}{lll}
c \beta & 0 & s \beta \\
0 & 1 & 0 \\
-s \beta & 0 & c \beta
\end{array}\right]
$$

The rotation angle $\gamma$ around $Z$ is expressed in equation (9).

$$
R(z, r)=\left[\begin{array}{ccc}
c \gamma & -s \gamma & 0 \\
s \gamma & c \gamma & 0 \\
0 & 0 & 1
\end{array}\right]
$$

$S$ is the abbreviation of $\sin$ and $c$ being the abbreviation of $\cos$ [24].

(2) Establishment of mathematical model

A manipulator is a linkage system that connects a series of linkage mechanisms through joints to realize the free movement of the head end $[25,26]$. And the homogeneous coordinate transformation between joints can accurately represent the position information of each linkage. And according to the sequence as shown in Figure 3, the joints of the manipulator are numbered, and three adjacent mechanisms are selected for analysis, forming the transformation matrix between the adjacent links.

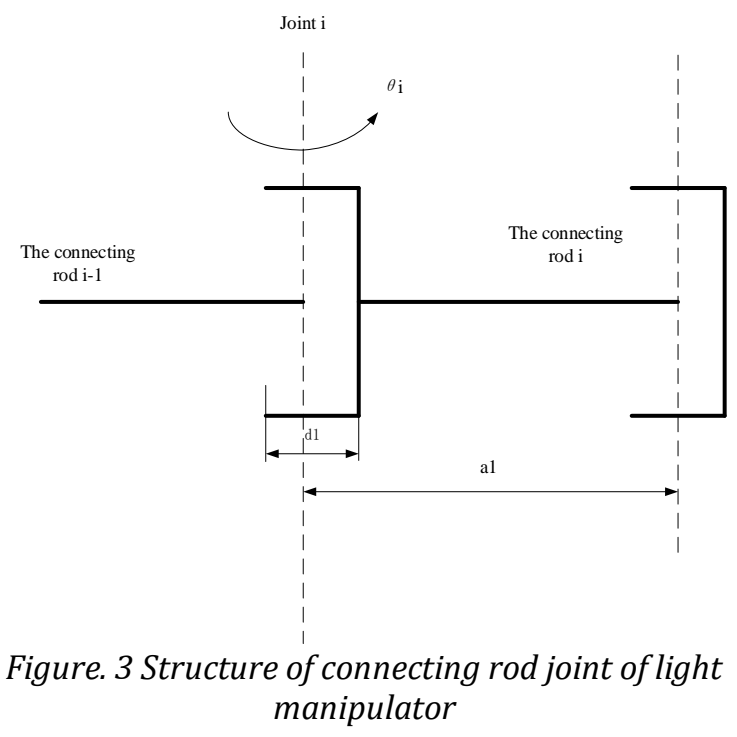

The mathematical model of light manipulator is established by setting parameters. The process is divided into the following two steps $[27,28]$.

(1) Coordinate system is established. Take the intersection point of the common vertical line and the axis of the joint $i-1$ and the two axes of the joint $i$ as the far point of the initial coordinate system $i$, and take the axis $i$ as $Z$ axis of the coordinate system $i$. Regard the discovery of the joint $i$ and axis $i+1$ as $X$ axis of the initial coordinate axis $i$, and make $Y$ axis of the coordinate system $i$ by the right-hand rule, which is the definition of the initial coordinate system. Similarly, the coordinate system $i+1$ and $i-1$ are defined in this way.

(2) D-H parameter is the characteristic parameter of linkage mechanism, which is defined as follows: the horizontal distance al from the axis $Z$ of $i$ to the axis $Z$ of $i+1$ along the axis $X$ is the length of the linkage based on the coordinate system $i$. The distance $\mathrm{d}$ of the linkage is from the axis $X$ of $i$ to the axis $X$ of $i+1$ along the axis direction $Z$. Around the axis $Z$, the angle from the axis $X$ of the coordinate system $i$ to the opposite direction of the coordinate system $i-1$ is the angle $\theta$ of the connecting rod under the coordinate system $i$. Correspondingly, the angle direction between the coordinate system $i+1$ and the connecting rod of $i-1$ is defined in this way.

\subsection{MATLAB Technology}

MATLAB is an interactive mathematical software developed by advanced programming language with algorithm development, data visualization, data analysis and numerical calculation. Because of its high-performance computing advantages, MATLAB is widely used in numerical analysis, numerical and symbolic calculation, engineering and scientific drawing, digital image processing, financial engineering. MATLAB uses matrix language as the basis of data analysis, including control statements, functions, data institutions and other complex data structures. And the object-oriented programming is realized on this basis. Users can input commands in the command window of the software, or execute a complex modified application. In the new version of MATLAB, C + + language is used, which makes it have a simpler way of data expression, combined with the data usage habits and writing format of scientific researchers, and convenient for non-computer researchers.

And the MATLAB program has the characteristics of portability and scalability, which is convenient for researchers to use in different systems. This also makes MATLAB widely used to solve mathematical problems in various fields, and provides comprehensive and effective solutions. It has the following advantages: 1 the software has excellent numerical calculation and symbolic calculation ability, which can change complex mathematical operation into simple data analysis process; 2) it has perfect graphics processing function, which can realize the visualization of 
calculation results and programming results; 3) stable application interaction interface makes the software have a more natural language close to mathematical expression, which is convenient to master and use; 4) MATLAB has a large number of application toolbox used in different research fields, such as signal processing toolbox, communication toolbox, etc., which provides users with a large number of convenient and practical processing tools [29].

\subsection{Design of Virtual Prototype based on ADAMS}

The steps of ADAMS virtual prototype are shown in Figure 4 and Table 1.

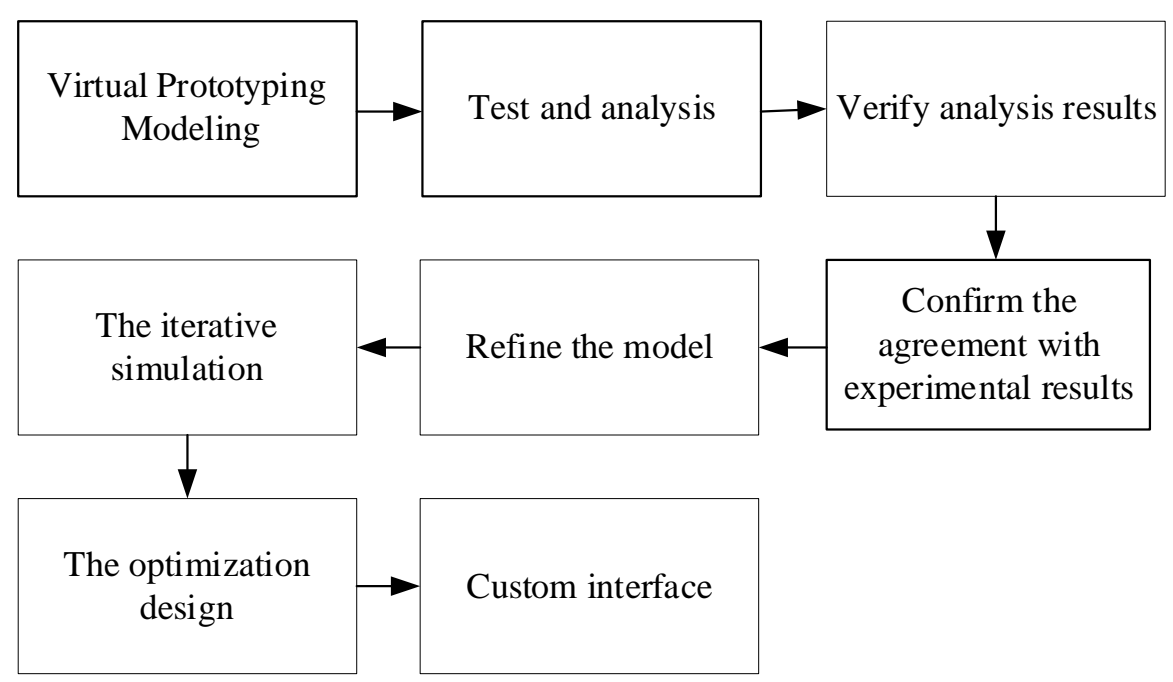

Figure. 4 ADAMS virtual prototype structure design process

Table 1 Steps of ADAMS virtual prototype

\begin{tabular}{|c|c|}
\hline Septs & Details \\
\hline 1. establish model & $\begin{array}{c}\text { Geometric modelling } \\
\text { Apply kinematic pair and constraint pair } \\
\text { Defining loads }\end{array}$ \\
\hline 2.. test and analyse the target & $\begin{array}{l}\text { Set test variables } \\
\text { Carry out simulation analysis } \\
\text { Replay the simulation process } \\
\text { Draw simulation data curve }\end{array}$ \\
\hline 3. test and analysis results & $\begin{array}{c}\text { Input test data } \\
\text { Add experimental data curve }\end{array}$ \\
\hline 4. optimization model & $\begin{array}{l}\text { Increase friction } \\
\text { Defining flexible bodies and connections } \\
\text { Define control } \\
\text { Modify the function of the applied force }\end{array}$ \\
\hline 5. simulation iteration & $\begin{array}{c}\text { Define design variables } \\
\text { Setting variable parameters }\end{array}$ \\
\hline 6. optimize the structure & $\begin{array}{l}\text { The main design factors were studied } \\
\text { The experimental design was studied } \\
\text { Carry out optimization research }\end{array}$ \\
\hline 7. customize the user interface & $\begin{array}{c}\text { Define user menu } \\
\text { Custom user dialog } \\
\text { Customize the above modelling steps }\end{array}$ \\
\hline
\end{tabular}

The following steps are needed to build the virtual prototype of ADANS: (1) the model. is built by using the database in ADAMS / View. The basic structure can be established through simple general modular parts. Other mainstream design software (CAD, PRO / E) can be used to model the light 
manipulator first, and then the data model can be imported into ADAMS software to supplement the constraints, motion trajectory and force needed by the model. (2) The model is tested. According to the actual application and the needs of the working conditions, the simulation experiment analysis and research of the established model can be carried out to verify the accuracy of the established model. (3)

The model is validated. The test data and simulation results are compared and analysed. (4) The model should be optimized. Optimize and refine the model according to the actual running track and actual working condition first, and add a series of more complex structure and operation mode to make the virtual prototype simulation model of case manipulator more applicable to the reality. (5) Simulation iteration is conducted. By repeating the feedback process, the desired goal or result approached gradually. In each iteration, the result is taken as the initial data value of the next iteration, which is carried out in the computer. Through the repeated subroutine (a group of instructions), the computer repeatedly executes the cycle in the program until a certain condition is met, and then stops the operation, which is also called iteration. Therefore, the concept of real-time dynamic variables of virtual model parameters is set first, and the iterative method is used to modify the parameter data, so that the best effect and the model simulation systematic are achieved. (6) The structure is updated. Compare and combine the simulation data with the actual conditions, and analyse and optimize the simulation model. (7) The user interface is customized. The user interface, the menu, user dialog box, question help and other practical application modules are made.

(1) Simulation example of manipulator trajectory planning based on MATLAB

Since designed trajectory is needed for the light manipulator to work in the actual condition, it needs to be simulated. The following is the actual trajectory of simulated welding. The weld trajectory is divided into arc type, straight line type and irregular curve type. Therefore, two trajectory planning schemes are designed: rectangular trajectory and elliptical trajectory. Rectangular trajectory is realized by trajectory planning method. The rectangular trajectory of the end of the manipulator is completely planned by using the translation matrix function transl 0 , Cartesian space trajectory planning function ctraj 0 , and inverse kinematics function ikine 0 . The ellipse trajectory depends on the joint trajectory planning method of the manipulator.
The joint space trajectory planning function JTRAJ $(0$ and forward kinematics function fkine $(0$ are used to plan the ellipse trajectory of the manipulator.

(2) Establishment of virtual prototype of light manipulator based on ADAMS

Firstly, the three-dimensional model of light manipulator is established, and the data of the model are imported into Adams system for analysis and processing. According to the actual needs, the solid assembly simulation of the light manipulator is carried out. Firstly, the basic parts (base, motor, gear, connecting rod) are created; secondly, the parts are assembled according to the assembly process. The assembly file is established in Pro / E and converted into Parasolid format to realize data transfer with ADAMS. Finally, the designed manipulator is simulated in ADAMS software, adjusting the torque and speed applied by the motor, conducting the dynamic and kinematic simulation of the manipulator motion data in different motion states, and analysing the actual performance of the manipulator through the animation motion effect of the model. For the parts with problems, the model can be returned to modify until the virtual design is completed. The prototype can meet the actual needs, and the engineering parameters of the manipulator are shown in Table 2, where $\mathrm{M}$ represents the weight in $\mathrm{kg}$ and $\mathrm{l}$ represents the length in $\mathrm{mm}$ [30].

Table 2 Technical parameters of construction manipulator

\begin{tabular}{|l|l|l|}
\hline $\begin{array}{l}\text { Component } \\
\text { name }\end{array}$ & Parameter & Value \\
\hline Forearm & M1 L1 & 42765 \\
\hline Middle arm & M2 L2 & 38700 \\
\hline Boom m & M3 L3 & 32825 \\
\hline
\end{tabular}

\section{Design and Simulation Results 3.1 Basic Structure of Light Manipulator and its Driving System}

The control system of the manipulator is complicated, and the main two parts are coordinated control and servo control. The basic structure of the virtual prototype and its driving system are shown in Figure 5. The structure of the manipulator includes five parts: gripper, small arm, middle arm, big arm, base and support. 


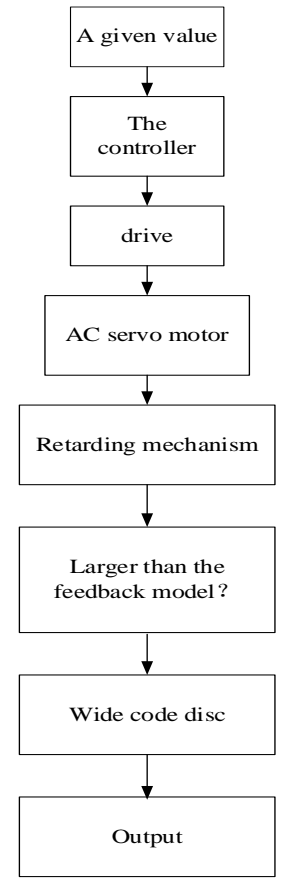

a)

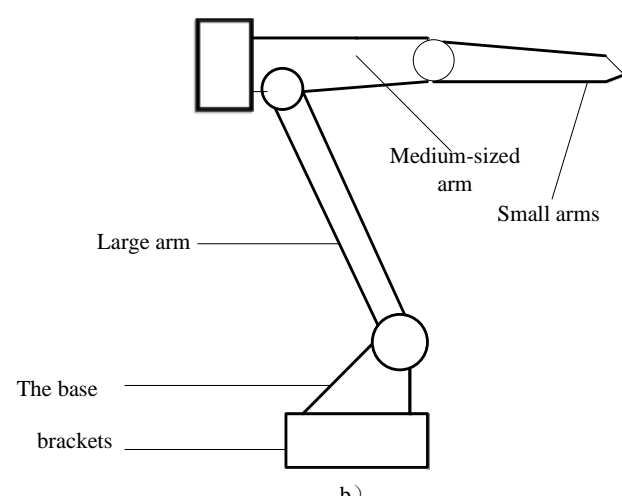

b)

Figure. 5 Virtual prototype structure and its driving system a) driving system B) manipulator structure

Coordinated control is through the computer and other peripheral equipment to control the machine for completing the tasks of receiving commands, coordinating control calculation, etc. Therefore, servo control involves the control of the whole system to control the effect each joint according to the pre-set trajectory. Therefore, the current industrial production and social life mostly use AC (alternating current) servo system.

The servo system of light manipulator is composed of the following mechanisms: controller, driver, driving motor, sensor, etc. The key motion parameters output by the coordination control layer are the input values of the servo system, and the key positions and speeds of the position.
The speed sensors are used as feedback signals. As for the difference between the given value and the feedback signal, the controller can adjust the control quantity. According to the control driver, the electric energy acting on the motor is different, so that it can run at different speeds. After the deceleration mechanism, the mechanical mechanism movement of each joint can be achieved.

\subsection{Virtual Prototype Simulation of Light Manipulator}

The displacement curve of each part of the $0 \sim$ 10 s manipulator is shown in Figure 6.
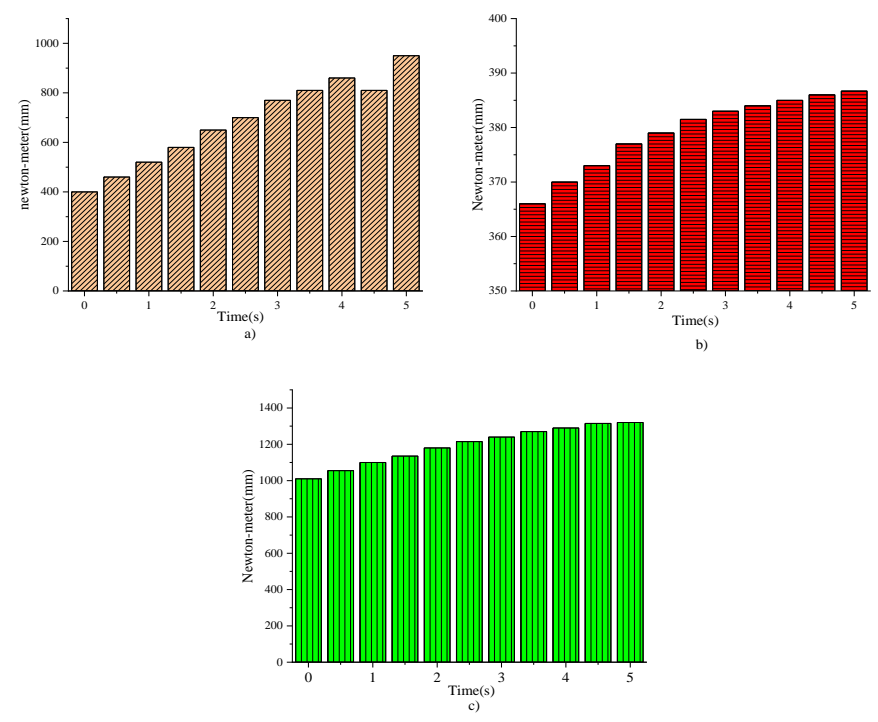

Figure. 6 Displacement of each part of light manipulator a) boom displacement b) middle-arm displacement C) forearm displacement 
In the process of $0 \sim 10$ s manipulator movement, when the time spent is 0 , it is at the starting position, with the speed of $0.427 \mathrm{~m}$, and when it is running for10s, it reaches the final position, with the speed of 0.277 according to the displacement curve (a) of the boom. In the displacement curve (b) of the middle boom, when the time spent is 0 , it is also at the starting position, with the speed of $0.298 \mathrm{~m}$, when it is running for $10 \mathrm{~s}$, it reaches the final position, with the speed of 0.198 ;
In the light of the displacement curve (c) of the hydraulic cylinder of the jib, when the time spent is 0 , it is at the starting position, with the sped of $0.298 \mathrm{~m}$, when it is running for $10 \mathrm{~s}$, it gets to the final position, with the speed of 0.198 The initial position is $0.358 \mathrm{~m}$, and the final position is 0.258 .

Figure 7 shows the torque value of the virtual prototype movement of light manipulator during the straight-line welding process

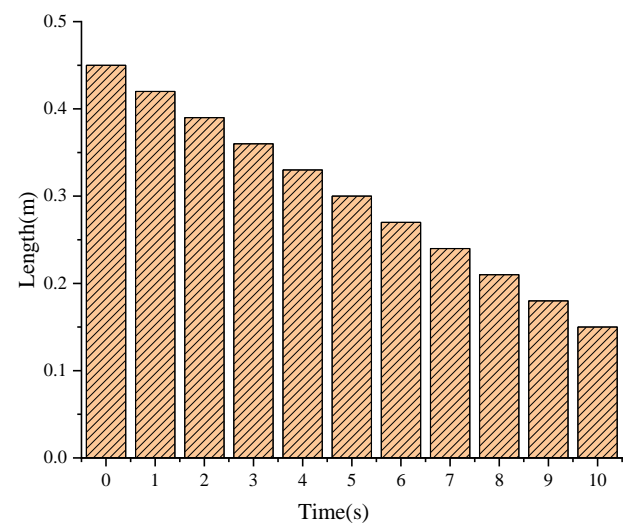

a)

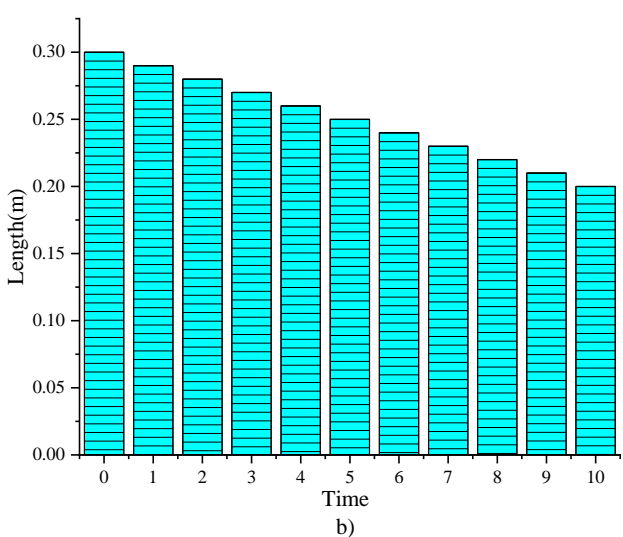

b)

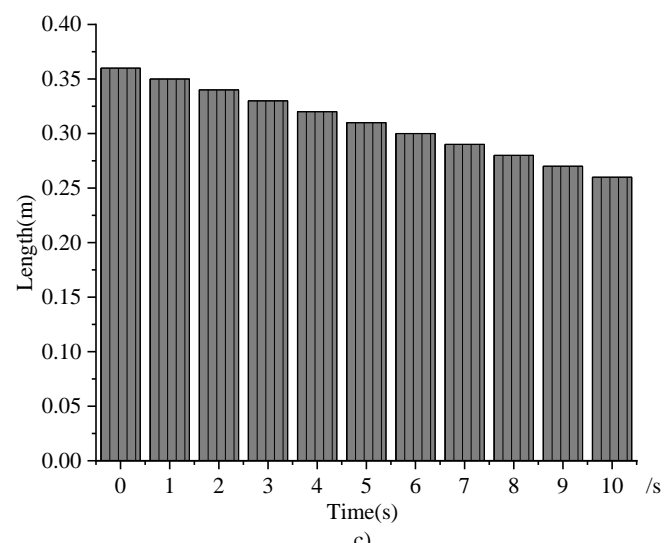

Figure. 7 Torque values of virtual prototype a) fore-arm torque b) middle-arm torque C) boom torque

The figure indicates that with the proceeding of the linear welding movement, the virtual prototype arm stretches out, the front end is far away from the base, and moves forward after 5 seconds. As shown in Figure 7, the torque of the virtual prototype increases with time runs, and the torque of the arm adjacent to the base is the largest, which increases from 1100 to 1300 , with a change of $30 \%$ in $0-5$ seconds. And the torque of the middle arm also changes with time, with a change from 366 to 390, increasing by $10 \%$.

The torque on the boom also changes with time, and it increases by $110 \%$, from 400 to 910 .

\subsection{Comparison and Verification of MATLAB Torque Calculation and ADAMS Virtual Prototype Simulation Structure}

In order to verify the validity of the designed dynamic model of the manipulator, the theoretical calculation value is introduced, the actual measurement value is compared, and the torque calculation value are analysed.

Firstly, the angle, angular velocity, angular acceleration and other values of the actual movement of the manipulator are derived from ADAMS software, and the torque of each arm of the manipulator is calculated by using MATLAB software. 
Moreover, the calculated torque value is obtained, and the measured and calculated torque values are

compared, showing in Figure 8.

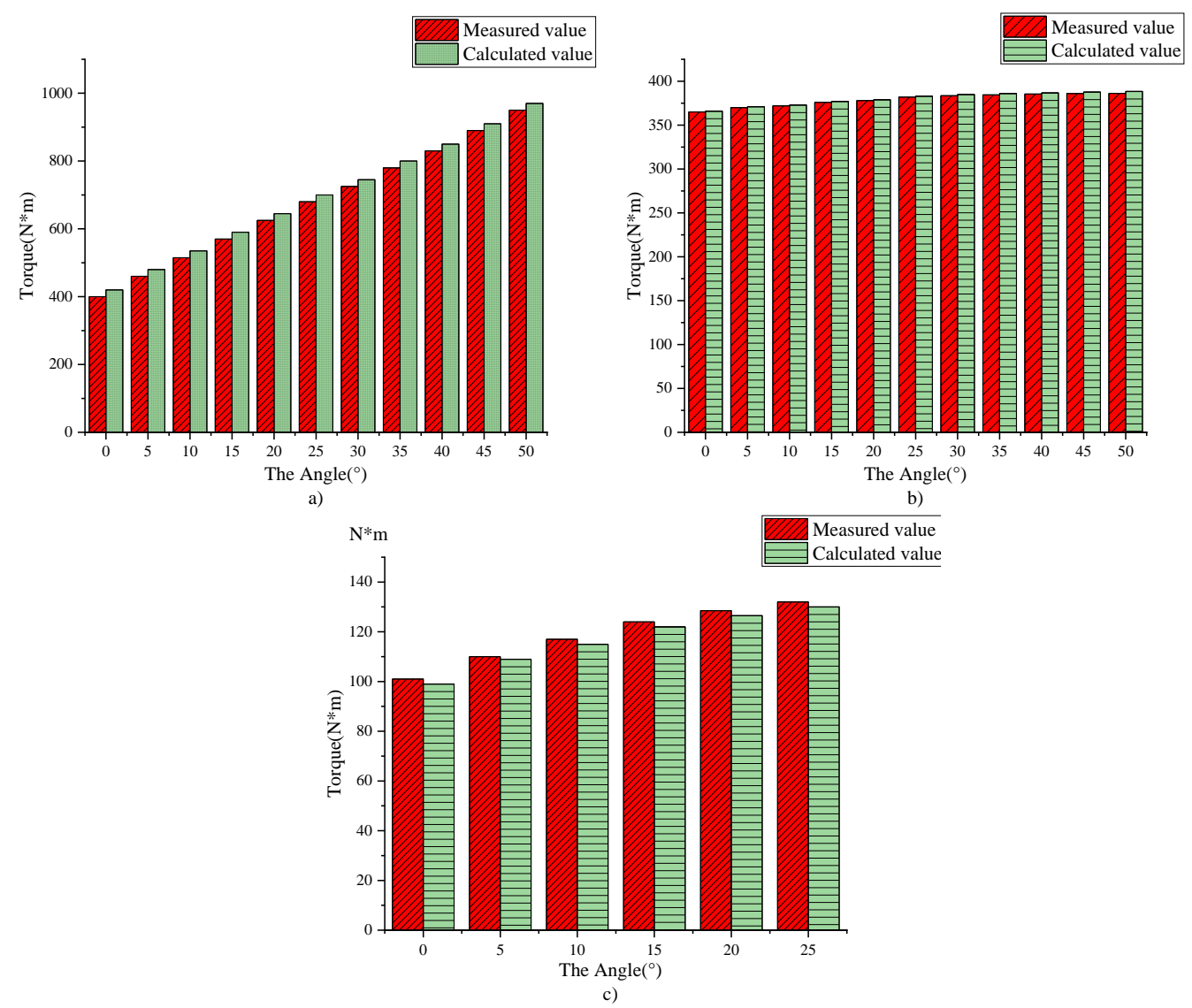

Figure. 8 Comparison of torque calculation value with measured value of virtual prototype a) forearm torque b) middle arm torque C) boom torque

According to (a), (b) and (c) in Fig. 8, the error between the torque calculation value and the actual measured value of the designed manipulator is within the allowable error range, which meets the requirements of the model simulation accuracy. The experimental results also show that the consistency of the dynamic model and the virtual prototype model is verified by this verification method.

\section{Conclusion}

A virtual prototype model for light manipulator of industrial machinery is established based on ADAMS software technology, and the feasibility of ADAMS technology to simulate the structure design of light manipulator is verified by actual measurement. Furthermore, the distribution law of torque value of the manipulator is calculated and the simulation results and actual results are compared.

The results show that the data of the prototype have a little difference from the actual need and the error is within the limit range. Therefore, the virtual prototype based on ADAMS technology can simulate the actual working conditions of light manipulator correctly, which will help with the future research on the structure design and improvement of light manipulator.

The following conclusions are obtained by the research of trajectory planning and control system design of light manipulator, as well as the comparison between the virtual prototype structure design and the actual data: (1) In the process of trajectory planning of manipulator, when the task of mechanical back has no path requirement, the trajectory planning of joint space and Cartesian space trajectory planning can be selected. However, in view of the minimum principle of flexibility and the joint space trajectory planning of the robot arm, when the movement path of manipulator is determined, Cartesian space trajectory planning can be used to plan the motion trajectory of the manipulator. (2) The virtual prototype model is built by ADAMS software, and the practical application simulation is carried out. The simulation results are similar to the actual needs. Therefore, the design of light manipulator based on ADAMS virtual prototype is successful.

Although this research generally achieves the research objectives and draws some valuable conclusions, there are still many deficiencies due to 
the limited academic experience. And the study may be limited in the following aspects: (1) the types of light manipulator are not developed, and many kinds should be analysed and compared. (2) the actual measurement data are insufficient to compare with the data obtained from virtual prototype, which will be done in the future research. The following aspects should be expected in the upcoming studies: (1) more simulation model of the light manipulator should be created to make it more diversified. (2) further understand the actual situation and obtain a large number of measured data. (3) update the structure of the light manipulator to make it competent for more complex conditions.

\section{References}

[1] Jain S., Gandhi A. (2021) Impact of artificial intelligence on impulse buying behaviour of Indian shoppers in fashion retail outlets. International Journal of Innovation Science, 1, 549.

[2] Andrew J. (2017) What Is Artificial Intelligence. Research World, 1(65), 75-75.

[3] Hassabis D., Kumaran D., Summerfield C. (2017) Neuroscience-Inspired Artificial Intelligence. Neuron, 95(2), 245-258.

[4] Larson C. (2018) China's massive investment in artificial intelligence has an insidious downside. Science, 41(2), 34-65.

[5] Pan Y. (2018) special issue on artificial intelligence 2.0: theories and applications. Frontiers of Information Technology \& Electronic Engineering, 34(2), 211-242.

[6] Sabatyan A., Rafighdoost J. (2018) Considerable diversity in generation light-arm beams using multi-twisted phase structure zone plate. Optics \& Laser Technology, 107(2), 24-31.

[7] Baghi R., Leila A. (2017) Differential Activation of the Dorsal Neck Muscles During a Light ArmElevation Task in Patients with Chronic Nonspecific Neck Pain and Asymptomatic Controls: An Ultrasonographic Study, PM\&R, 34(4), 122-145.

[8] Jiang L., Dou S. (2018) Characteristic parameter change of circuit breaker under closing spring fatigue. Transactions of the Canadian Society for Mechanical Engineering, 4(2):411-443.

[9] Yoskovitz S., Shaul G. (2019) AUTOMATIC MECHANICAL SYSTEMS DIAGNOSIS. Transactions of the Canadian Society for Mechanical Engineering, 2(1), 671-831.

[10] Xu F., Jiang Q., Rong L. Structural model and dynamic analysis of six-axis Cartesian coordinate robot for sheet metal bending[J]. International Journal of Advanced Robotic Systems, 2019, 16(4):172988141986156.
[11] Davis L. (2018) AFENET Evaluation Word cloud evaluation design matrix laboratory strengthening. The Pan African medical journal, 30(1):297-298.

[12] Smither S., Eastaugh L., Filone C. (2018) TwoCenter Evaluation of Disinfectant Efficacy against Ebola Virus in Clinical and Laboratory Matrices. Emerging Infectious Diseases, 24(1), 32-59.

[13] Dai Y., Yamashita N. (2017) Convergence analysis of sparse quasi-Newton updates with positive definite matrix completion for twodimensional functions. Numerical Algebra Control \& Optimization, 1(1):61-69.

[14] Baruzzo G., Hayer K., Kim E. (2017) Simulationbased comprehensive benchmarking of RNA-seq aligners. Nature Methods, 14(2), 135-139.

[15] Salamandra K., Tyves L. (2017) Integral principle in the problems of dynamic analysis of gearshift in automatic gearboxes. Journal of Machinery Manufacture \& Reliability, 46(5), 434441.

[16] Park K., Kim B., Kim H D. (2017) Carbon Fiber Preform Automatic Layer Mold Desk Design Stability Study by Dynamic Analysis. Ksmpe Conference. 12(5), 343-371.

[17] Dupre D., Andelic N., Morrison G. (2018) Dynamic Analysis of Automatic Facial Expressions Recognition 'in the Wild' Using Generalized Additive Mixed Models and Significant Zero Crossing of the Derivatives. British HCI, 3(7), 454-477.

[18] Herrero S., Pinto C., Altuzarra O. (2018) Analysis of the 2 P RU-1 P RS 3DOF parallel manipulator: kinematics, singularities and dynamics. Robotics \& Computer Integrated Manufacturing, 51(9), 63-72.

[19] Xanthidis M., Kyriakopoulos K., Rekleitis I. (2018) Dynamically Efficient Kinematics for Hyper-Redundant Manipulators.23(1):43-63.

[20] Qu H., Guo S. (2017) Kinematics analysis of a novel planar parallel manipulator with kinematic redundancy. Journal of Mechanical Science and Technology, 31(4):1927-1935.

[21] Li M., Xu T., Foerste C. (2019) Improving the Performance of Multi-GNSS Ambiguity Fixing for Airborne Kinematic Positioning over Antarctica. Remote Sensing, 11(8), 992.

[22] Madson A., Fielding E., Sheng Y. (2019) HighResolution Spaceborne, Airborne and In Situ Landslide Kinematic Measurements of the Slumgullion Landslide in Southwest Colorado. Remote Sensing, 11(3), 122-152.

[23] Hilker M., Richtler T., Barbosa C E. (2018) The Hydra I cluster core - II. Kinematic complexity in a rising velocity dispersion profile around the galaxy, 32(5), 441-452. 
[24] Scheidl J. (2020) Motion of a friction belt drive at mixed kinematic description. International Journal of Solids and Structures, 2(1), 200-201.

[25] Dese M., Galera M., Aylton P. (2019) GPS Satellite Kinematic Relative Positioning: Analyzing and Improving the Functional Mathematical Model Using Wavelets. Mathematical Problems in Engineering, 2(1), 266-287.

[26] Sheng Y. (2017) Mathematical Model for the Effect of Dynamic Parameter Posed on Free Floating Space Manipulator's Kinematic Accuracy Advanced Materials Research, 216(2),254-260.

[27] Kimberly D., Adam M. (2017) An Inverse Kinematic Mathematical Model Using Groebner Basis Theory for Arm Swing Movement in the Gait Cycle. J Symbolic Computation, 202(2), 259 332.
[28] Prada E., Kelemen M., Gmiterko A. (2020) Locomotive, principally kinematic system of snakelike robot mathematical model with variable segment length International Conference on Mechatronics. Mechatronika, 19(1), 255-292.

[29] Kumar K., Srinath A., Harish M. (2017) SIMULATION OF FOUR ARM PARALLEL MANIPULATOR FOR MEDICAL APPLICATIONS. Journal of Advanced Research in Dynamical \& Control Systems, 9(18):1802-1809.

[30] Albalasie A., Hussain I., Horoub M. (2019) Design, Prototype, and Control Design Based on Computed Torque Control of Selective Compliance Assembly Robot Arm. Annual International Conference on CYBER Technology in Automation, Control, and Intelligent Systems, 9(1), 5(1), 32-61. 